\title{
El castillo de Iznájar y las reformas castellanas de Pedro I (1362- 1366)
}

The castle of Iznájar and the castilian remodelling of king Pedro I (1362-1368)

\section{Ángel Rodríguez Aguilera}

Gespad al-Andalus SLU, Granada, Spain, angelrodri@gespad.com

\begin{abstract}
This paper presents a unique case of castilian remodelling of an islamic fortress, the castle of Iznájar, conquered by Pedro I between 1362 and 1366, with the clear intention of staying in the territory. For that purpose, defensive improvements were introduced, wich are clearly identified in the context of the fortified compound. This episode, well bounded in time, allows to recognize the Castilian contributions to the Nasrid defensive achitecture.
\end{abstract}

Keywords: Military architecture, fourteenth century, the border of the Fingdom of Granada, Castle of Iznájar.

\section{Introducción}

Iznájar es una población del extremo sur de la provincia de Córdoba, muy próxima al Poniente granadino, localizada en la margen derecha del rio Genil. De hecho, históricamente era una de las ciudades del antiguo reino nazarí de Granada que por los avatares de la historia, especialmente de la reconquista, administrativamente quedó dentro de la provincia de Córdoba.

El núcleo urbano ocupa una de las últimas estribaciones meridionales del sistema subbético cordobés. La población se encontraba amurallada, existiendo una gran alcazaba en la parte más alta, que es lo que hoy se conoce como la villa y el castillo. Realmente se trata de la misma unidad espacial, en la que se llevó a cabo una segregación a finales del siglo XIV para garantizar la defensa de un reducto más pequeño. Este fenómeno tiene que ver con la propia historia del sitio y el avance de la frontera castellana hacia el sur.

La investigación del castillo de Iznájar se ha realizado en varias fases, ligada a la restauración, promovida por el Ayuntamiento de Iznája con el apoyo del Ministerio de Fomento.

\section{Contexto histórico}

Independientemente de la posibilidad de que el emplazamiento del castillo ya fuera ocupado durante la Antigüedad, como evidencian las tumbas excavadas en la roca que existen al pie de la Torre del Homenaje, los restos arqueológicos y las primeras referencias históricas claras nos indican que la principal fase de ocupación es la de época medieval. El castillo de Iznájar aparece citado por primera vez en el contexto de las revueltas de la fitna del siglo IX d.C. El topónimo Hisn Ashar, nos indica un punto fortificado de escasa entidad, con una población estable de muladíes.

En un primer momento se relaciona con la rebelión de Umar ibn Hafsun (Vallvé Bermejo, 1965), del que tenemos como referencia tanto un texto de Ibn Hayyan (Martínez Antuña, 1937, pp. 109- 


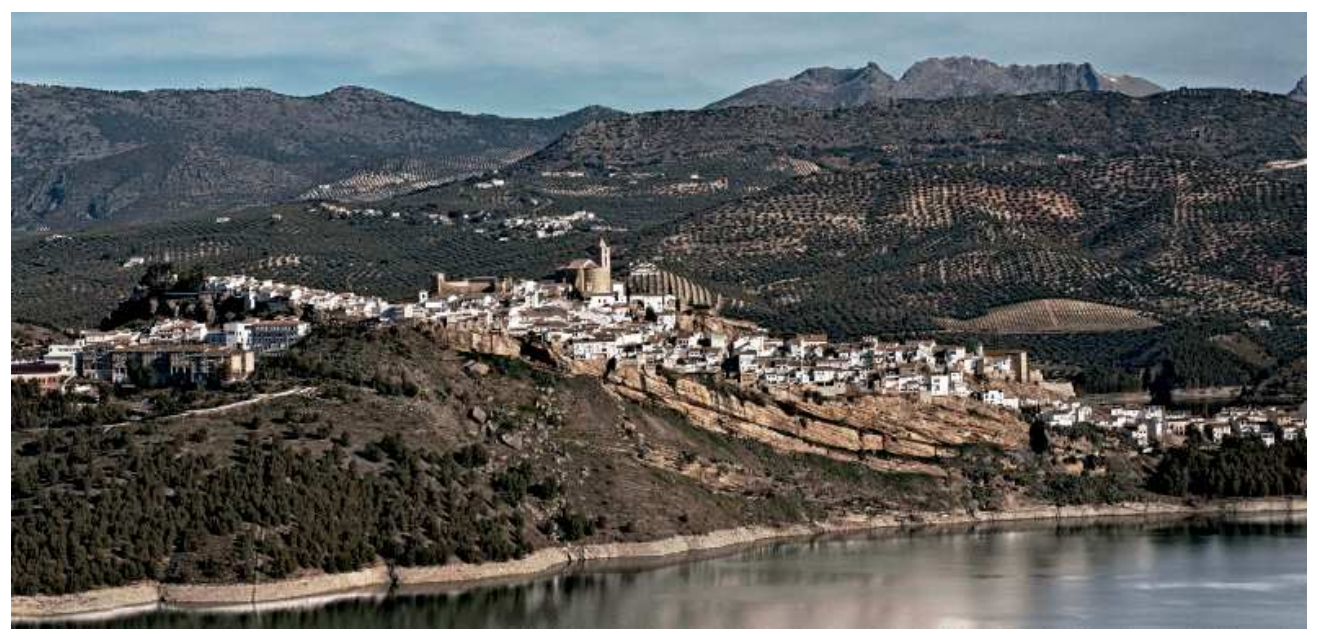

Fig. 1. Vista general de la población de Iznájar sobre el río Genil.

110) como la Crónica de ${ }^{c}$ Arib sobre al-Andalus. En este texto se apunta que en el 910-911 d.C. la fortaleza estaba bajo el control de Fadl b. Salama, yerno de Sa'id b. Mastana, "no obstante los habitantes de esta fortaleza, al objeto de ganarse el afecto del imam 'Abd Allah, le dieron muerte, y varios de ellos se presentaron con su cabeza en la Bab al -Sudda [de Córdoba], acción que se les agradeció" (Castilla Brazales, 1992, p. 108).

Antonio Arjona Castro, en una serie de trabajos (Arjona Castro, 1976, 1979, 1999) analizó en varios trabajos el topónimo e interpreta las escasas noticias sobre Iznájar en época emiral y califal. Este periodo abarca desde el 844 al 911, año en el que definitivamente Iznájar, acata el dominio de Córdoba (Arjona Castro, 1999). A partir de esta época las referencias no son muy abundantes pero sí que nos permiten apuntar en cierta medida la evolución histórica del emplazamiento. Así, en el siglo XI es citado como uno de los castillos cuyo territorio es incorporado desde primera época en el reino zirí de Granada (Lévi-Provençal, García Gómez, 1982, p. 85) Desde el punto de vista territorial, por su posición, al sur del macizo de las Sierras Subbéticas y su proximidad a la ciudad de Archidona, Iznájar pertenecía a la cora de Rayya.

A partir de este momento las fuentes árabes guardan silencio sobre el castillo, solo citado en el siglo XII por Idrisi como "fortaleza que contiene una población muy numerosa y en donde hay un zoco muy frecuentado" (Rodríguez Aguilera, 2013, p. 18). Aparece de nuevo en el contexto de la conquista del valle del Guadalquivir en el siglo XIII, especialmente en las fuentes cristianas. En época nazarí mantiene su importancia como plaza estratégica del extremo occidental del reino, controlando a su vez un distrito administrativo. De hecho, ibn al-Jatib, en la Lamha, los cita como el décimo catorce distrito del sultanato de la siguiente forma: "El clima de Iznájar, en el que está el castillo de Niwalis" (Ibn al-Jatib, 1998, p. 17).

Iznájar queda como castillo de primera línea fronteriza en el lado granadino integrado en el área de la ciudad de Loja (Malpica Cuello, 2014, pp. 257, ss.).

A principios del siglo XIV Alfonso XI, en el contexto de asegurar el territorio de la subbética cordobesa, hizo el primer intento de conquista, fallido por las inclemencias meteorológicas:

"Et el Rey tobo / cercado doce dias el logar de Rute, et los Moros entregarongelo. Et quisiera ir cercar á Isnajar que tenian los Moros; et por quanto era llegado el mes de setiembre, et facia grandes aguas, et otrosí era cumplido el tiempo que avian á servir los Ricos-omes et Caballeros que fueran con él, et él non tenia de que les dar sueldo, dexó de ir cercar aquel Isnajar, et fue a la torre de Matrera” (Cerdá y Rico, 1787, p. 469). 


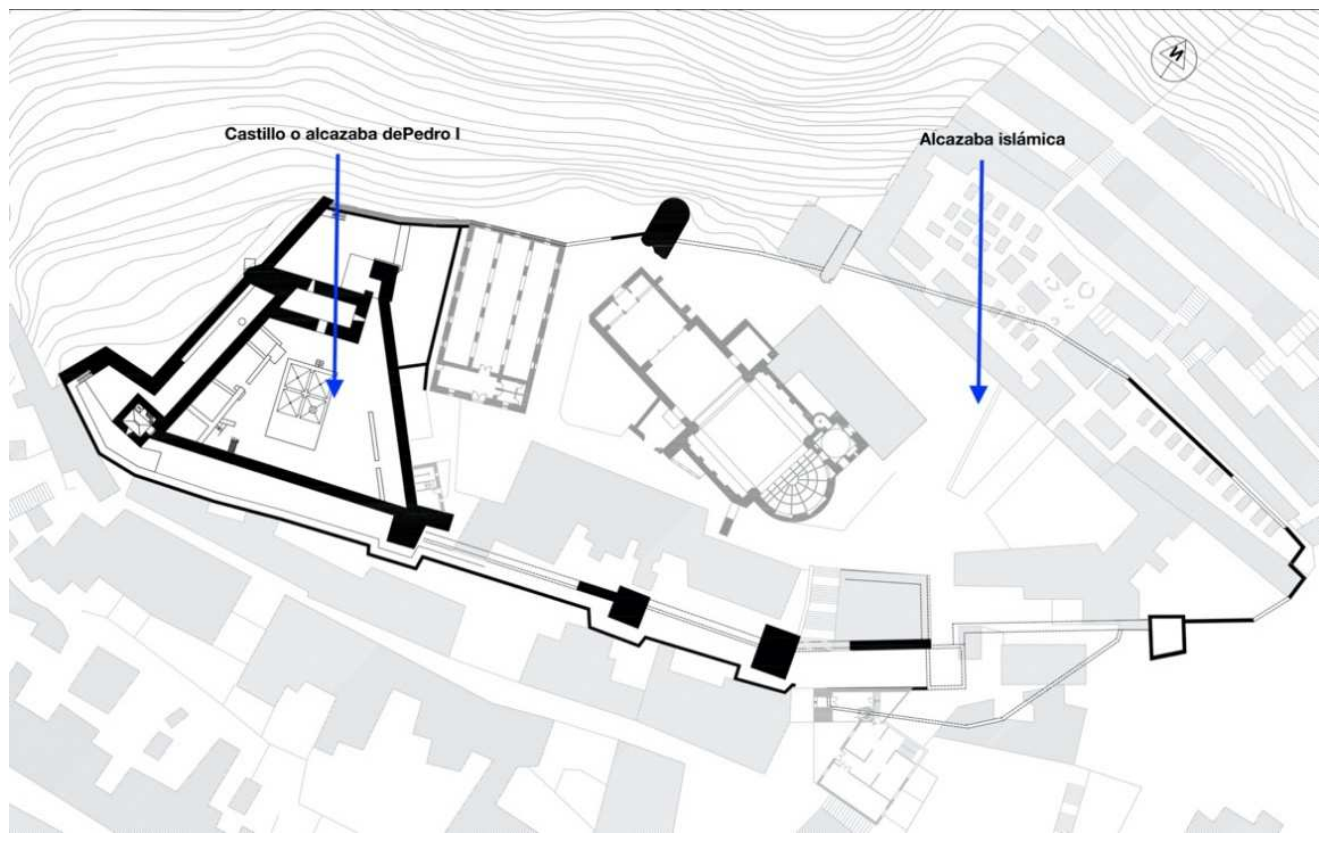

Fig. 2. Planta general de la alcazaba y castillo de Iznájar.

Esta referencia nos permite contextualizar el papel que juega Iznájar en el marco de la frontera en la época de Alfonso XI que pasa por ser, con el de Fernando III el Santo, uno de los periodos más fructíferos en la expansión cristiana por el valle del Guadalquivir. El acontecimiento, fechado en 1339, es previo a la gran victoria del Salado, de 1340 sobre los ejércitos meriníes y nazaríes, que consolida el avance hasta el mismo borde septentrional y algunos valles internos de las Subbéticas cordobesas. Su importancia estratégica se multiplica y así queda reflejado en las crónicas islámicas durante el reinado Muhammad V, coetáneo con el de Pedro I el Cruel. Tras el breve paréntesis de los años 1362-1366 en el que estuvo bajo control directo de las huestes de Pedro I, la villa fortificada fue conquistada definitivamente por los castellanos en 1431, integrándose desde entonces en el mayorazgo de la casa de los Fernández de Córdoba.

\section{Breve descripción del castillo de Iznájar}

El castillo de Iznájar ocupa la parte más elevada del cerro en el que se asienta el casco histórico de la villa. La planta actual es producto de la evolu- ción histórica y se nos presenta de forma triangular, insertado en el conjunto de la población fortificada Su tipología corresponde con el de "villa de frontera" nazarí (Malpica Cuello, 2000), en la que es posible diferenciar la alcazaba del resto del recinto, que adquiere un cierto sentido urbano, cuyo desarrollo está en relación con el proceso histórico experimentado. Ejemplos similares y que ilustran este aspecto los encontramos en los paralelos de las villas de Moclín, Íllora y Montefrío, todas del lado granadino.

En el caso de Iznájar, es de especial interés el conjunto de la alcazaba del que se segrega el recinto del castillo propiamente dicho ya que muestra importantes reformas y modificaciones acometidas en el breve periodo que estuvo bajo poder de las tropas de Pedro I, y que supusieron una transformación en el concepto y funcionalidad del hisn islámico para transformarlo en un baluarte de resistencia en primera línea de frontera. Por tanto, la realidad material con la que nos encontramos como punto de partida es compleja. La descripción detallada se ha realizado comenzando por las estructuras ubicadas al norte del recinto y se ha seguido el sentido de las agujas del reloj. Se han individualizado las los lienzos y torres, para luego 
identificar espacios o ámbitos funcionalmente coherentes.

En un primer nivel descriptivo diferenciamos tres ámbitos:

1) El castillo propiamente dicho. De planta triangular, formado por los Lienzos L02, 03 y 04, las Torres T01, 02, 03, 04 y 05; y por el edificio de Pedro I (E01). En su interior alberga, además de la citada construcción, un aljibe, un silo-mazmorra, la Torre del Homenaje y un patio de armas rodeado por un conjunto de viviendas.

2) Barbacana. Construcción fechada en el periodo de reformas arquitectónicas en el que el castillo estuvo en manos de las tropas de Pedro I. Está formado por un conjunto de lienzos que son los L06, 07, 08, 09, 10, 11, 12 y 13. Conserva, además del alzado de sillares, el camino de ronda, el peto de protección construido en tapial en el que se abren una serie de aspilleras, los sistemas de drenaje, una poterna de salida al exterior, y las escaleras de comunicación entre los distintos tramos de antemurro. Dicho elemento llega hasta la torre pentagonal en proa (T05), creando varios recorridos estancos. Al noroeste de dicha torre quedan las evidencias del arranque de otro tramo de barbacana que quedó abortado por la reconquista nazarí de 1366.

3) Sistema de acceso. El hecho de reducir el espacio de la alcazaba para ocupar solo un sector susceptible de ser defendido con garantías obligó a crear un nuevo acceso, aprovechando una antigua torre albarrana para crear una entrada en un complejo de torre con paso acodado y angosto, defendido desde la vertical que conecta con el edificio de Pedro I.

4) Recinto de Muhammad V. Denominamos de esta forma el recinto que queda al norte del conjunto y que viene a rematar el cierre septentrional de la barbacana inconcluso por los cristianos. Sirve a su vez de zona de tránsito y comunicación con el resto de las fortificaciones de la villa. A este periodo también se le adscribe las reformas de mejora y reparos tras el asalto, acondicionándolo de nuevo para la función defensiva de la frontera.

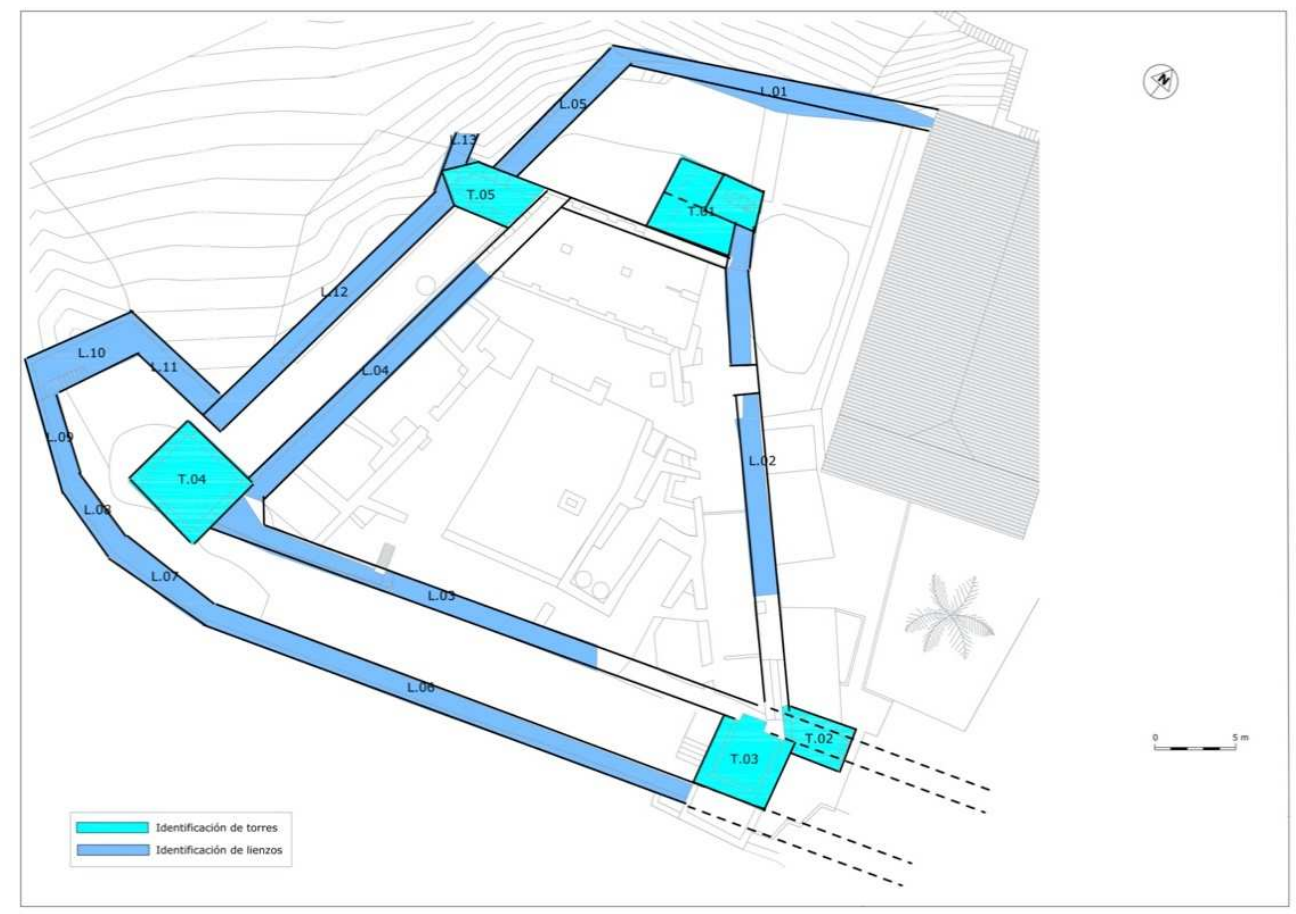

Fig. 3. Planta general del recinto del castillo con identificación de lienzos y torres. 


\section{La conquista de Pedro I y la recuperación de Muhammad V}

Las relaciones que se establecieron entre los reyes Muhammad V y Pedro I hay que insertarlas en un momento de crisis interna tanto en Granada como en Castilla (Fernández Puertas, 2018). El sultán Muhammad V, que accedió al trono tras la muerte de su padre Yusuf I en 1354, es el artífice de la consolidación del reino nazarí (Ali Makki, 1995). Consolidación no sólo de sus fronteras, sino también de su estructura como "estado" y de la cultura nazarí. Debido a su temprana entronización, su largo reinado estuvo sometido a periodos de inestabilidad interna como el que le llevó a ser depuesto del trono entre 1359 y 1362 por Muhammad VI el Bermejo.

Por su parte, Pedro I tuvo que hacer frente a la guerra por los derechos al trono planteada por su hermanastro Enrique II de Trastámara.

Ambos monarcas se apoyaron mutuamente frente a sus adversarios y si bien el nazarí acabó imponiéndose a Muhammad VI, la muerte del castellano en 1369 propició el cambio dinástico. En este contexto, en 1362, año en el que Muhammad $\mathrm{V}$ recuperó el trono, los castellanos ganaron la plaza de Iznájar entre otras pero, se asentaron de forma estable en ésta. Es posible que la pasividad de los nazaríes por recuperarla tuviera que ver con que el hecho se produjo siendo aún rey Muhammad VI y la ocupación del castillo pactada con Muhammad V. Se mantuvo bajo control castellano hasta 1366, momento en el que el nazarí se siente libre del pacto con Pedro I, acuciado por la guerra en Castilla y con Aragón.

En esos cuatro años, entre 1362 y 1366, se acometieron importantes obras que apuntan a una clara voluntad de permanecer en el territorio y convertir a Iznájar en una punta de lanza que con facilidad permitía alcanzar la Vega de Granada remontando el surco Intrebético. Esta afirmación tiene su correspondencia con los cambios sufridos en la estructura de la alcazaba: se acotó un espacio más reducido, de planta triangular, para garantizar su defensa, y se reforzó de forma muy notable con fábrica de cantería.

Del mismo modo, tras la reconquista nazarí de 1366 fue necesario reparar las brechas abiertas en los muros y reordenar las defensas diseñadas por los castellanos, concluyendo su programa defensivo con un diseño mucho más pobre materialmente y de forma más precipitada. No obstante, se mantuvo la organización espacial diseñada entre 1362 y 1366.

Para entender el alcance de las reformas de los dos momentos contamos con relatos históricos y la materialidad de castillo. Los primeros son fundamentales a la hora de identificar con claridad las distintas fábricas y asignarles una cronología bastante precisa.

El análisis visual de los restos constructivos conservados del castillo permite diferenciar la existencia de una fábrica muy homogénea y propia en el estilo de las construcciones castellanas góticas. Se trata del uso de cantería muy bien cuidada y del uso de diseños ya experimentados en otras fortalezas reconstruidas en época de Alfonso XI, como la de Alcalá la Real -conquistada definitivamente en 1341- que se reconocen en Iznájar. Estos paralelos, junto con la información histórica nos permite caracterizar la fase constructiva asociada al periodo en el que la fortaleza estuvo en manos castellanas.

El relato que nos proporciona la correspondencia diplomática de Muhammad V también es clara al respecto:

"Iznájar es un poste inaccesible, una fortaleza notable y un tormento evidente. El infiel se dirigió a ella cuando su firme resolución le dio su dominio y su preocupación el deseo de ocuparla, y cerró y reparó sus brechas, atendió a sus partes débiles, ampliando sus fortificaciones hasta el extremo de cortar e interceptar todas las ambiciones y deseos (respecto de la posesión de la fortaleza)" (Gaspar Remiro, 1914, pp. 305-307)

Las obras atribuibles a los castellanos son las siguientes:

\subsection{Lienzo de muralla L02}

Para garantizar una mejor defensa del espacio que ocuparon las huestes de Pedro I, optaron por crear un espacio aislado dentro de la alcazaba. Para ello, reaprovechando la torre del Homenaje y los lienzos norte, sur y oeste, construyeron otro que acotaba el flanco oriental, creando una planta 
triangular. Esta muralla inicialmente se construyó con sillarejo de tamaño mediano, muy regular y bien dispuesto en hiladas, con sistema de evacuación de aguas hacia el foso exterior y la propia alcazaba.

En el interior encontramos dos elementos muy relevantes.

\subsection{Edifico castellano}

Edificio de sillares que cierra el castillo por el norte y que nosotros hemos venido a denominar como Edificio de Pedro I. Funciona como recinto estanco o Torre de Homenaje desarrollado en planta baja y terraza, con un diseño y arquitectura que evidencian una vocación claramente defensiva. De planta trapezoidal $(9,7 \mathrm{~m}, 3,24 \mathrm{~m}, 129 \mathrm{~m}$ y $3,7 \mathrm{~m}$ ), y cubierto con una bóveda de cañón ligeramente apuntada, presenta un aspecto macizo e inexpugnable, refugio de las tropas cristianas. El muro de fachada tiene un grosor de $1,75 \mathrm{~m}$ y un hueco de acceso de 1,30 m de anchura que conserva las huellas para encajar el alamud que cerraba por dentro la sala. El muro de cierre por el Este presenta una pequeña ventana de aireación, sin función militar, en la parte alta mientras que en el extremo opuesto, se adosa directamente contra la muralla islámica. El muro norte es el que presenta mayor complejidad ya que si bien su fábrica es igual a la descrita, se sirvió de la muralla existente en algunos puntos, siendo demolida y sustituida en otros, reaprovechando una torre pentagonal, a la que se adosa, para utilizar defensivamente su terraza que se asoma sobre dos tramos de barbacana y se proyecta hacia la defensa de la vertical exterior.

También se vincula estructuralmente y funcionalmente a la torre 01 que formó parte del acceso fortificado diseñado en el periodo 1362-1366, convirtiéndose en torre-puerta.

En conjunto, los sillares están dispuestos en hiladas, con una altura media de $0,26-0,28 \mathrm{~m}$ y se utilizan piezas muy homogéneas de 0,30-0,40 m, alternando ocasionalmente con otras de hasta 0,5 $\mathrm{m}$. El mortero utilizado es de cal y las juntas entre los sillares están selladas de esta forma.

La parte alta queda coronada por una azotea que conserva el peto original de tapial, con almenas hacia el norte. La argamasa empleada, de árido y cal, presenta menos compacidad que la islámica e introduce en su composición un porcentaje mayor de materia orgánica y restos de artefactos (cerámica). El encofrado suele ser de 0,4 $\mathrm{m}$ de espesor y las tongadas, o lechadas, de $0,20 \mathrm{~m}$. El peto tenía unos $0,8 \mathrm{~m}$ altura y los merlones eran de dos tipos: rectangulares, de $1,20 \mathrm{~m}$ de altura por 0,70 de anchura, y escalonados, o dobles. En el flanco norte todavía son visibles uno completo del primer tipo y la mitad del segundo además de una batería de tres aspilleras abiertas en el peto que defendían el acceso a la puerta y que fueron cegadas en una época posterior. L hueco de selección de tiro es de 0,44 m de altura por 0,20 de anchura.

El acceso a este punto se hacía desde la planta alta de la torre-puerta y desde el adarve que conectaba con la Torre del Homenaje. No tenía conexión con el interior del edificio.

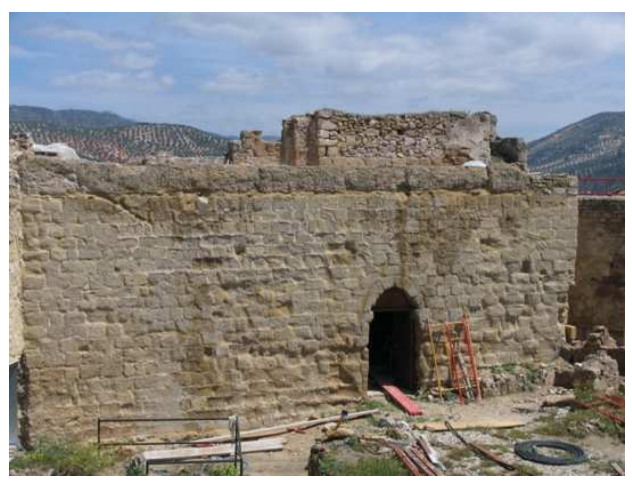

Fig. 4. Vista general del edificio, con puerta de acceso y remate con peto de tapial.

La construcción de este volumen queda bien definido cronológicamente como parte de las obras emprendidas por Pedro I aunque su funcionalidad puede prestarse a varias hipótesis. En principio, por sus características de defensa pasiva, actúa como el principal espacio de reclusión segura de las tropas cristianas, si bien también pudo actuar como capilla o iglesia. Ibn al-Jatib refiere el derribo de las campanas, que podrían haber formado parte de un espacio de culto que con toda probabilidad existió. El único edificio que pudo albergar esa función es éste, pudiendo haber estado colocadas en la azotea, desde la que se controla visualmente el entorno. Existen paralelos similares 
en castillos del sureste, como en el de Socovos (Albacete), entregado a principios del siglo XIII a la Orden de Santiago y en el que se construyó un edificio muy parecido en su planta y que si se identifica claramente con la capilla del castillo (Simón García, 2010, p. 359).

\subsection{La torre-puerta (torre T01)}

Reconstrucción de una torre albarrana islámica y su transformación en puerta en codo, además de refuerzo de la planta superior, con un pequeño cuerpo al que se accede por medio de escaleras y que permite comunicarse con el adarve y la terraza del edificio. La cara norte está formada por la unión de dos muros de distinta época: uno de mampostería en hiladas marcadas entre ripios y alternando el aparejo en espiga, fechado en época islámica, al que se le adosa por medio de una interfaz de unión, otro de sillares, levantado por los castellanos. Éste tiene una altura mayor y ambos en conjunto se cohesionan funcionalmente como la torre $\mathrm{T} 01$.

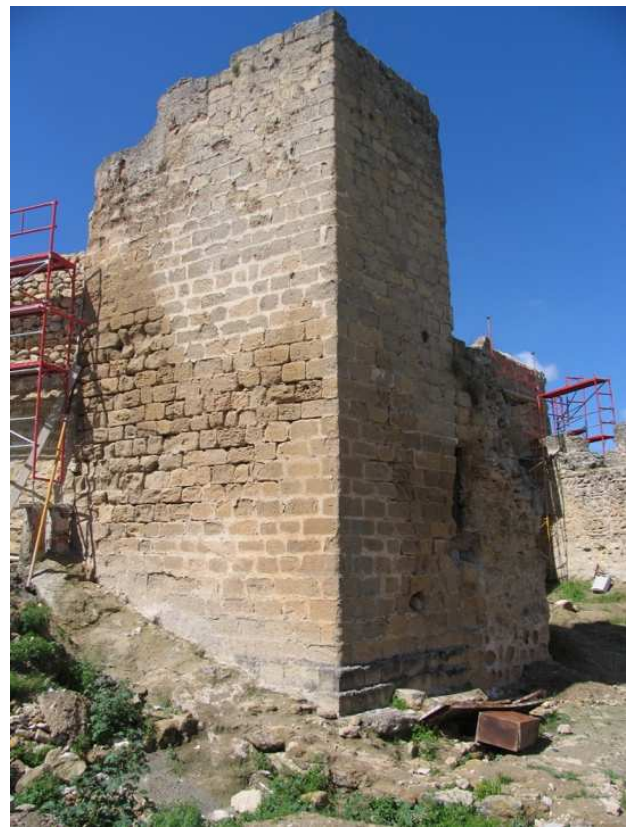

Fig. 5. Torre 01. Torre-puerta. Fábrica castellana. Vista exterior.

Su cimentación está formada por una zarpa escalonada, visible por el exterior. En el lado sur se repite esta dualidad de fábricas, con una parte islámica de sillares que queda separada de la castellana por una interfacies en esquina muy bien definida. La torre cierra por el Este con un muro similar al de sillares, del que arranca el Lienzo L02, con remate de tapial en su coronación. Interiormente está cubierto con una bóveda de cañón en la que también se aprecian claramente dos fases constructivas: en el cuerpo islámico queda el arranque de la cubierta original, construida con sillarejo de tendencia cuadrada, probablemente el puente de conexión de la torre albarrana, mientras que el resto es una reconstrucción de 1362 utilizando lajas de calcarenita dispuesta verticalmente, como en la Sala de Pedro I.

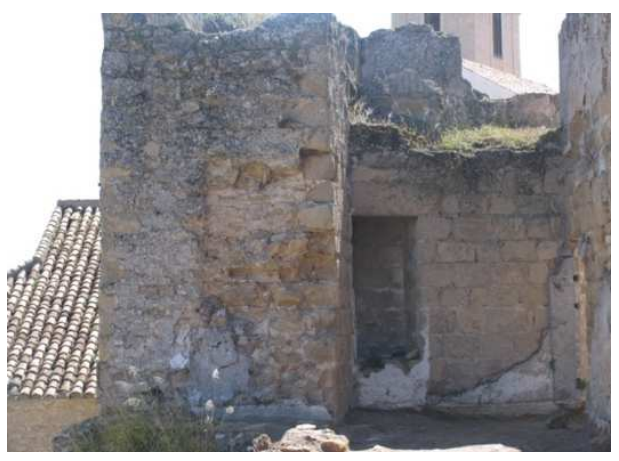

Fig. 6. Torre 01. Torre-puerta. Planta superior.

\subsection{Antemural o barbacana}

La defensa del reducto castellano se completó incorporando un antemuro, que rodeaba los lados sur y este, quedando abortado su desarrollo desde la torre pentagonal en proa. También en uno de sus tramos incorpora una poterna Se trata sin duda del elemento más significativo y emblemático de esta fase. Es una construcción de sillares, situada por delante de la muralla islámica con camino de ronda y peto de tapial construido y que permite crear una doble línea defensiva. El espacio entre ambos, la liza, estuvo exento a modo de foso muy profundo con circulación seccionada por compartimentos estancos. Este elemento es uno de los recursos defensivos más usuales en la arquitectura militar de Península Ibérica a partir del siglo XII. Normalmente se le denomina barbacana, aunque según L. de Mora-Figueroa es un término mal 
aplicado a este tipo de construcciones (Mora Figueroa, 2006). La barbacana es uno de los elementos más característicos de las reformas defensivas emprendidas por las tropas de Pedro I, entre 1362 y 1366 . Se trata de un antemuro con vocación de abrazar la primitiva alcazaba. Y decimos con vocación porque sólo llegó a edificarse en su flanco sur y oeste, es decir en la parte más próxima al reducto del castillo, quedando abortado su desarrollo por el norte debido a la conquista de Muhammad V. Por el lado sur alcanza hasta la puerta de entrada a la alcazaba, y queda separa del resto por la torre 03 .

Ciñéndonos al castillo se identifican tres sectores defensivos diferentes:

Sector 1: Tramo de la barbacana formado por el antemuro y la liza en el extremo sur del castillo. Arranca de la base de la torre T03, y formado por los Lienzos L10, L11, L12 y L13, protege el flanco sur de la Torre del Homenaje. Conserva en la base el sistema de evacuación de agua del foso. También el sistema de acceso al adarve por medio de una rampa de tapial que monta sobre la base de la torre 03 . El adarve tiene un empedrado basto, sobre el que arranca el peto de tapial con batería de saeteras, sin paradós. La circulación inferior en la liza queda cortada por la roca sobre la que cimenta la Torre del Homenaje.

Sector 2: Tramo formado por un reducto más amplio, en la base de la Torre del Homenaje, formado por los lienzos L09 y L08, sirviendo de transición en altura y en el espacio hacia el extremo oeste. No se conecta con el adarve interior, interrumpiendo la circulación superior.

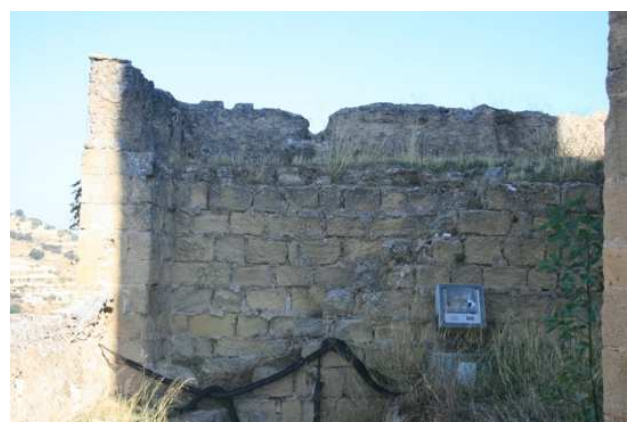

Fig. 7. Detalle del antemuro, paramento interior, en el sector 2 .
Se accede por medio de una escalera de cantería. La anchura del adarve es mayor y también está rematado con peto de tapial.

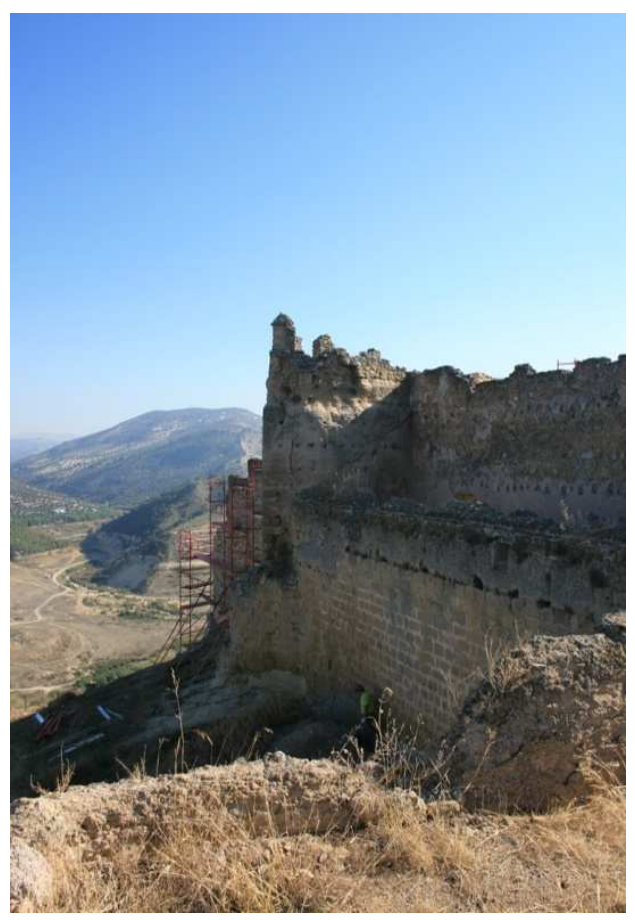

Fig. 8. Vista exterior del antemuro y de la torre pentagonal.

Sector 3: Tramo de la barbacana formado por el antemuro y la liza del flanco oeste del castillo, que con el Lienzo L07 llega hasta la base de la Torre pentagonal.es el más homogéneo, con una liza con una poterna de sillería con dintel de piedra y dos molduras semicirculares en la coronación de las jambas.

\section{La reconquista nazarí y el proceso de re-for- tificación}

La restitución en el trono de Muhammad V en 1362 y el cambio que se dio en la guerra civil castellana, propició la reconquista de Iznájar en 1366. Afortunadamente nos ha llegado una información muy interesante, procedente de fuentes de carácter epistolar diplomático y por el propio ibn al-Jatib. 
La carta enviada al sultán de Fez es especialmente interesante por la cantidad de información que aporta:

"Iznájar es un poste inaccesible, una fortaleza notable y un tormento evidente. El infiel se dirigió a ella cuando su firme resolución le dio su dominio y su preocupación el deseo de ocuparla, y cerró y reparó sus brechas, atendió a sus partes débiles, ampliando sus fortificaciones hasta el extremo de cortar e interceptar todas las ambiciones y deseos (respecto de la posesión de la fortaleza). Había en ella un crecido número de tiradores de teas y tizones encendidos, y no hay que decir del número de sus armas y de aprestos. Toda ella estaba ajustada a la necesidad y preparada para la violencia, a no contar con Dios cuyo poder es invencible y cuya bravura son incapaces de rechazar todas las multitudes infieles. La embistieron los muslimes precipitándose entre las olas del martirio, corriendo a porfía hacía las líneas de la muerte y entregando generosamente las almas puras en medio de la proclamación de Dios. Entonces fue ganada la meseta contigua a la fortaleza y los defensores que había en ella corrieron a resguardarse en el interior de aquélla, perseguidos por los muslimes. Ésta fue la primera señal del triunfo y el comienzo de la victoria prescripta.

Los muslimes atacaron aquella altura deseada en los flancos de la fortaleza, abrieron extensas brechas en sus muros, obstruyeron anchas partes de sus fosos profundos y resistieron con un valor de que no pueden dar brillante idea el pensamiento ni la imaginación. Como que solamente ello es uno de los vestigios de la Providencia divina, con los cuales son cumplidas las promesas en favor de la religión muslim y por medio de ellos Dios muestra su gracia en bien del Islam.

Consideraron los enemigos cuanto era el espanto que causaba en sus espíritus el poder de Dios y se confundieron sus inteligencias, se deshincharon las heridas entre ellos, les flaquearon las fuerzas y convinieron en pedir la amnistía después de excusarse y de llegar en su resistencia hasta el mayor extremo. Fueron correspondidos en su propósito y se les cumplió el deseo, y descendieron en la forma más abigarrada, como de un campo de las estrellas. Sobre las torres de la fortaleza fueron enclavadas las banderas del Islam, fue ensalzada la palabra de la Unicidad, fueron purificadas las casas de los siervos y su posesión fue interpretada como modelo.” (Gaspar Remiro, 1914, p. 305)

También relatado en la carta al sultán de Túnez:

"Entonces nos retiramos de aquel castillo, luego de haber proveído a su defensa y guarnición y al acrecentamiento de sus provisiones y de trabajar por nuestra mano en la reparación de las brechas producidas por el combate y de las aberturas practicadas en el interior por los golpes de los sables de los peones" (Gaspar Remiro, 1914, p. 358).

Quizás una de las poczas fortalzas que sin duda tienen elementos construidos que pueden ser atribuidos a la voluntad de Muhammad V es la de Iznájar. Del análisis espacial de la fortaleza se desprende una serie de reformas posteriores a las de Pedro I que cierran el circuito defensivo del castillo. Éstas deben asociarse a este momento y son las siguientes:

1) Reconstrucción del almenado y peto sobre la muralla.

2) Reparaciones en la muralla sur.

3) Cierre del circuito de barbacana cristiana por el tramo norte, con la construcción de los lienzos L05 y L01 utilizando mampostería en hiladas.

4) Reparaciones de la torre-puerta.

\section{Bibliography}

Ali Makki, M. (1995). "La Granada nasrí", in Arte islámico en Granada. Propuesta para un museo de la Alhambra, Granada, pp. 41-54.

Arjona Castro, A. (1976). "La cora de Córdoba", Actas del I Congreso de Historia de Andalucía, Córdoba, vol. I, pp. $27-46$.

Arjona Castro, A. (1979). "El castillo de Turrush", Boletín de la Real Academia de Córdoba de Ciencias, Bellas Letras y Nobles Artes, 49, 100, pp. 257-264. 
Arjona Castro, A. (1999). "Iznájar en la Historia de Al-Andalus”, in Primeras Jornadas de la Real Academia de Córdoba sobre Iznájar, Córdoba, pp. 213-216.

Castilla Brazales, J. (1992). La Crónica de c Arib sobre al-Andalus, Impredisur, Granada.

Cerdá y Rico, F. (1789). Crónica de d. Alfonso el Onceno de este nombre de los reyes que reynaron en Castilla y León, Madrid, in http://bibliotecadigital.jcyl.es/i18n/consulta/registro.cmd?id=5309 (15 January 2020).

Fernández Puertas, A. (2018). Alhambra. Muhammad V, Almed Ed., Granada.

Gaspar Remiro, M. (1914). “Correspondencia diplomática entre Granada y Fez. Siglo XIV”, Revista del Centro de Estudios Históricos de Granada y su Reino, Tomo IV, Granada.

Ibn al-Jatib. (1998). Historia de los reyes de la Alambra, Molina, E.; Casciaro, J.M., eds., Granada.

Ibn Hayyan. (1937). Al-Muqtabis fi Tar'rij riyal al-Andalus -al-qism al-talit-, Antuña Ed., Paris.

Lévi-Provençal, E.; García Gómez, E. (1982). El siglo XI en 1ª persona. Las “memorias” de Abd Allah, último rey zirí de Granada, destronado por los Almoráviedes (1090), Alianza Editorial, Madrid.

Malpica Cuella, A. (2000). "Las villas de frontera nazaríes de los Montes granadinos y su conquista", in Barrios Aguilera, M.; González Alcalntud, J.A., Las Tomas. Antropología histórica de la ocupación territorial del Reino de Granada, Diputación de Granadas Ed., Granada, pp. 33-136.

Malpica Cuello, A. (2014), Las últimas tierras de al-Andalus. Paisaje y poblamiento del reino nazarí de Granada, Ed. Universdiad de Granada, Granada.

Mora-Figueroa, L. de. (2006). Glosario de arquitectura medieval defensiva, Madrid.

Rodríguez Aguilera, A. (2013). El castillo de Iznájar. Un castillo nazarí en el reino de Córdoba, Ayuntamiento de Iznájar.

Simón García, J.L. (2010). Castillos y torres de Albacete, Instituto de Estudios Albacetenses “don Juan Manuel” Ed., Albacete.

Vallvé Bermejo, J. (1965). “De nuevo sobre Bobastro”, Al-andalus, 30, pp. 137-174. 